\title{
The effect of interfacial properties and liquid flow on the stability of powder islands
}

\author{
Xin Yi Ong ${ }^{1, *}$, Spencer E. Taylor ${ }^{2}$, and Marco Ramaioli ${ }^{1}$ \\ ${ }^{1}$ Department of Chemical and Process Engineering, University of Surrey, United Kingdom GU2 7XH \\ ${ }^{2}$ Department of Chemistry, University of Surrey, United Kingdom GU2 7XH
}

\begin{abstract}
This study aims at understanding the interplay between the interfacial properties of the powder grains and the characteristics of the liquid flow used to disperse them, in order to obtain an effective dispersion of a powder in a liquid, avoiding air entrainment. The dispersion of grain "rafts" and powder islands "stacks" was investigated both on a static and on a moving air-liquid interface. Powder wicking prevents the formation of a powder island when the grain contact angle is below a critical contact angle. Above the critical contact angle, a powder island forms and grows to a critical depth that depends on grain radius and contact angle. Imposing a flow on the air-liquid interface can either promote water impregnation, reducing the depth of the powder island or destabilise the whole island. In the latter case, the island sinks, forming a heterogeneous powder structure that is wet outside and dry inside.
\end{abstract}

\section{Introduction}

A typical example of dispersion of powders in liquids is the reconstitution of beverages from dehydrated powders. Minor changes in the physicochemical properties of the powder can affect dramatically the outcome of this process $[1,2]$. Understanding the interplay of grain surface properties (especially contact angle) [3-5] density, particle size [1], addition rate of beads to the interface $[6,7]$ and liquid flow fields is necessary in order to enhance wettability. When a cylinder or a sphere is at air-liquid interface, stability is governed by forces arising from their mass, capillarity and buoyancy [8].

The question of how an object sinks has drawn much scientific interest. As a result of increased object density or wettability, or decreased surface tension, the weight of an object exceeds the vertical restoring balancing force created by surface tension and the hydrostatic force [9]. Vella \& Li [10] proposed the influence of contact line effects may be an important factor during the transition process from floating to sinking. There is a critical impulse speed below which the object is trapped by the interface and floats, or above which it pierces the interface and sinks [6, 7, 9].

During wettability studies, Jurin's theory [11], Washburn's law [12] have been widely employed to explain the process of liquid wicking in a porous medium e.g. layered beads $[12,13]$. However, there is a significant difference when wicking occurs in cylindrical capillaries and in a powder beds consisting of spherical particles. The powder bed has indeed varying pore cross section and solid-liquid orientation, which affect the flow behaviours [14, 15]. Several groups [2, 16-18] showed that the critical contact angle, $\theta_{0}{ }^{*}$, below which wicking takes place in monodisperse layers is with the range between $51^{\circ}$ and $85^{\circ}$, considerably lower than $90^{\circ}$ limit for cylindrical capillaries. Jones et al. [19] discussed how the deposition geometry of powders affects the size limits of the assemblies before they sink through the interface by comparing the critical size that "rafts" [20] and "stacks" [2] can achieve. A raft is formed by depositing new spheres away from the spheres that are already at the air-liquid interface, causing the spheres to self-assemble through interfacial deformation and gravity, this being termed the "Cheerios effect" [20]. A stack is formed by the deposition of new spherical grains on top of grains that are already floating at the interface [19]. Raux et al. [2] studied the creation of a powder island (equivalent to a stack) using grains with the contact angle, $\theta>\theta_{0}{ }^{*}$ and showed that wicking occurs when the stack depth, $h$, exceeds a critical stack depth, $h^{*}$. Several authors $[19,21,22]$ have used the ratio of the density of the deposited particles to those of the surrounding fluids to compare the depth of interfacial deformation triggered by the presence of the particles to the capillary length, i.e.

$$
\Delta=\frac{\left(\rho_{S}-\rho_{B}\right) r_{0}}{\left(\rho_{B}-\rho_{A}\right) a}
$$

where $r_{o}$ is the radius of the particle, $a$ is the capillary length $(\gamma / \rho g)^{1 / 2}, \gamma$ and $\rho$ are the surface tension and density of the liquid, and $g$ is the gravitational acceleration. Through equation (1), these authors found that the maximal density ratio, $\Delta_{\max } \approx 1$, allows both raft and stack geometry to remain afloat.

Consistency with most studies cited above, in this study insoluble grains are used to reduce the complexity

* Corresponding author: x.ong@surrey.ac.uk 
of the system. The contact angle below which wicking is observed on a static interface is measured for different grain sizes. The effect of liquid flow in the vicinity of the point of impact of the grains is then discussed.

\section{Experiments}

Different glass bead diameters, $d_{p}$ were used in this study: $<0.106,0.212-0.300,<0.50$ and $0.75-1.00 \mathrm{~mm}$. Bead density, $\rho_{s}$ was $2500 \mathrm{~kg} / \mathrm{m}^{3}$ and the beads were silanised by treatment with a $5 \%$ dichlorodimethysilane solution in $n$-hexane to change their surface properties to hydrophobic, following the literature protocol proposed by Hamlett et al. [17]. The bead contact angle, $\theta$ was then tuned by changing the surface tension of the wetting liquid, using mixtures of deionised water (DI) containing different ethanol volume fractions, $V_{f}$. The contact angles of the silanised glass beads were measured by depositing them gently onto the air-liquid interface of different solutions, in a $1 \mathrm{~cm}$ cuvette (Fig. 1). Each measurement was repeated on ten different beads.

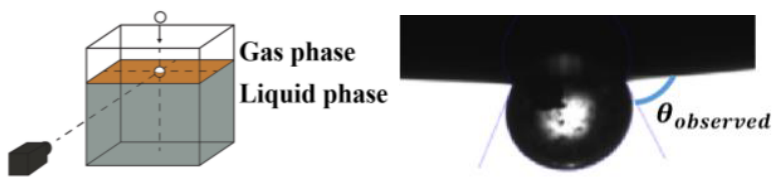

Fig. 1. Contact angle analysis in a cuvette.

The powder islands were created by pouring the beads gently onto a static air-liquid interface from a paper funnel located $30 \mathrm{~mm}$ above the initial liquid surface. A raft was initially formed, followed by a central stack (Fig. 2). The evolution of the beads at the liquid-grain interface was recorded using a Basler camera (acA1929$155 \mu \mathrm{m})$. The average mass flow rate for each size of bead is $0.12 \pm 0.01 \mathrm{~g} / \mathrm{s}$. The dimensions of this "static cell" were: $15.5,11.0,8.0 \mathrm{~cm}$.

A purpose-built "flow cell" was also used, to investigate how the liquid flow affected the stability of rafts and stacks formed at the air-liquid interface. The dimensions of this cell are indicated in Fig. 3. Once a stack had been created, the impellers were rotated at 3.3 rev/s in centre-down direction, depicted in Fig. 3.

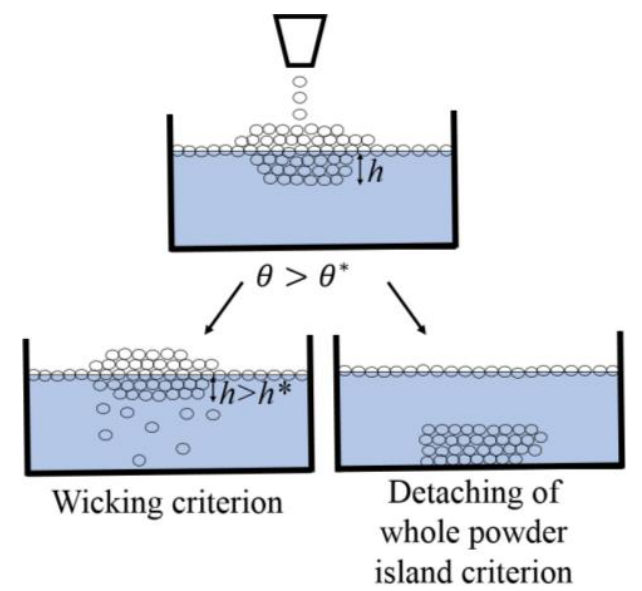

Fig. 2. Experimental setup to investigate the powder islands formed at static air-liquid interface. Wicking (bottom left) and island detachment (bottom right) were alternatively observed.

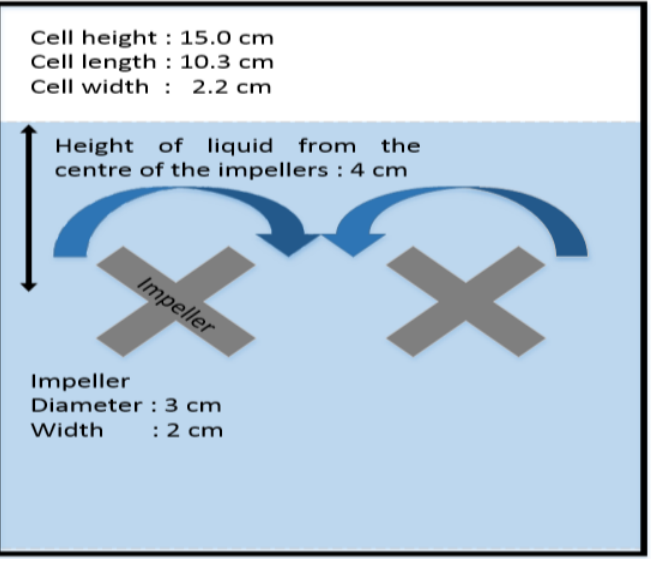

Fig. 3. Experimental setup to investigate powder islands formed at air-liquid interface with a moving fluid.

\section{Results}

\subsection{Contact angle}

As shown in Fig. 4, water-ethanol mixtures allow the grains to show contact angles varying from $98 \pm 1.5^{\circ}$ to $57 \pm 3.9^{\circ}$ as $V_{f}$ increases from 0 to 0.5 . As $V_{f}$ increases, surface tension decreases, which leads to decreasing contact angle.

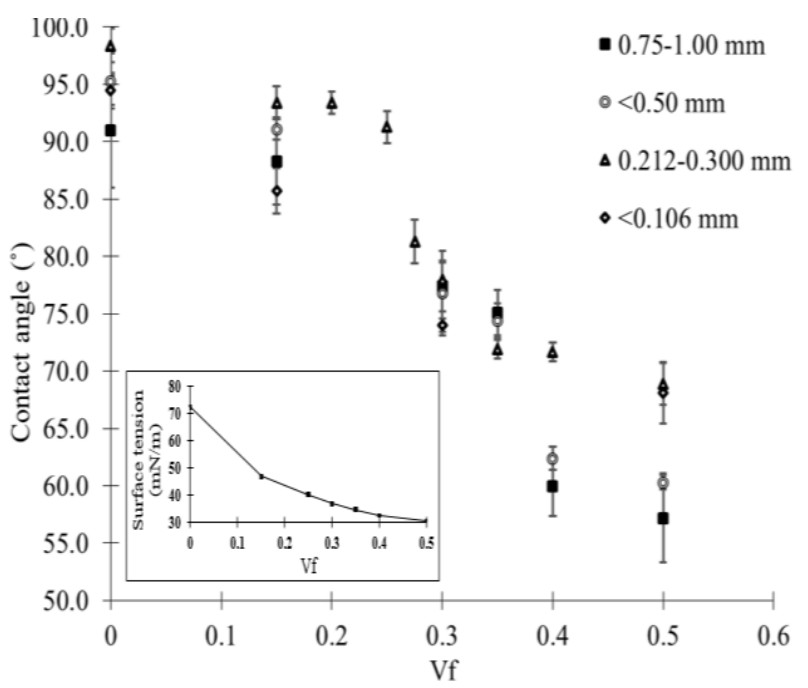

Fig. 4. Effect of the liquid ethanol volume fraction, $V_{f}$ on the contact angle of silanised glass beads of different diameter, $d_{p}$ (indicated in the legend). The inset shows corresponding values of surface tension of different $V_{f}$.

\subsection{Static cell}

The top row of Fig. 5 shows pictures of single beads floating at the static interface of different water-ethanol mixtures. The bottom row of Fig. 5 shows pictures of the powder island (stacks) built using the same grains and $V_{f}$. These pictures were taken just before the onset of wicking and were used to measure the critical stack depth, $h^{*}$ above which the liquid wicks in the pores of these stacks of grains. The grain size was kept constant $\left(d_{p}=0.75-1.00 \mathrm{~mm}\right)$ and the contact angle decreased with increasing $V_{f}$. This in turns resulted in a strong decrease of the critical stack depth. When $V_{f} \geq 0.40$, 
which corresponds to $\theta<72^{\circ}$, the system can only support rafts instead of stacks for bead size ranging from $<0.106$ to $1.00 \mathrm{~mm}$.

The critical stack depths are summarised in Fig. 6, separating the non-wicking from wicking or detachment of the whole stacks. As described in Raux et al. [2], when the stack depth is increased, the liquid-air interface in the pores between the beads at the bottom of the stack becomes curved, due to the liquid hydrostatic pressure, $p$ $=\rho g h^{*}$, inducing a curvature $\mathrm{C}=\rho g h^{*} / \gamma$ and this favours wicking. In this study, the critical stack depth is found to be somehow dependent on the bead size. Smaller beads show a higher critical stack depth, as shown in Fig. 6. The critical stack depths were fitted with straight lines, having slopes of respectively $30,54,28,68 \mathrm{~mm} / \mathrm{rad}$ for $d_{p}(\mathrm{~mm})=0.75-1.00,<0.50,0.212-0.300$ and $<0.106$.

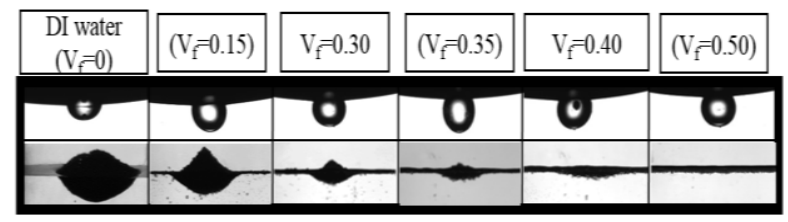

Fig. 5. (Top) Silanised glass beads of diameter $d_{p}=0.75-1.00$ $\mathrm{mm}$ placed at the air-liquid interface with different ethanol volume fractions, $V_{f}$. (Bottom) Critical stack depth, $h^{*}$, formed at different $V_{f}$.

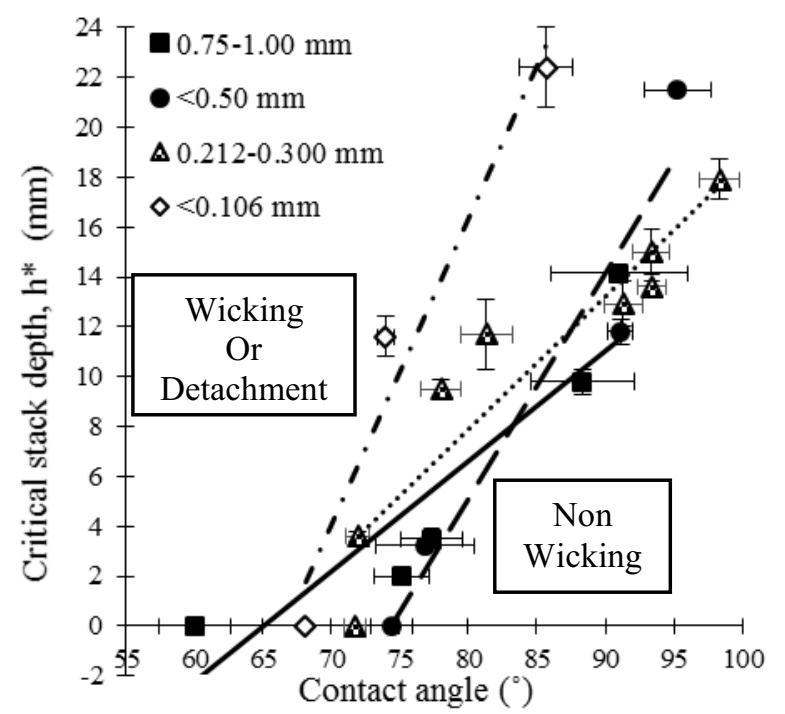

Fig. 6. Effect of the bead contact angle on the critical stack depth. Beads of different diameter are represented using different symbols, as described in the legend.

The theory developed by Raux et al. [2] describes the critical stack depth, $h^{*}$ formed with bead having contact angle $\theta>\theta_{0}{ }^{*}$ :

$$
h^{*}=\frac{4 a^{2}}{d_{p}} \frac{\cos \theta_{0}^{*}-\cos \theta}{\cos ^{2} \theta_{0}^{*}}
$$

where the critical contact angle to obtain a stack composed by two layers is $\theta_{0}{ }^{*} \approx 51^{\circ}$. Fig. 7 shows that the normalised critical stack depth, $h^{*}$ decreases fairly linearly with $\cos \theta$, in qualitative agreement with the theory of equation (2). However, the slope predicted by the model is much larger than the value that can be obtained by the fitted straight line on the experimental slope, as illustrated in Fig. 7 and Table 1. Raux et al. [2] also found a similar inconsistency. The causes of this inconsistency are currently being investigated. Wicking is not always observed when adding grains to an existing stack, as illustrated in Fig. 8, the detachment of the whole stack can also occur. Wicking is observed at the bottom of the stack constructed from larger beads, $d_{p}$ $(\mathrm{mm})=0.75-1.00$ and $<0.50$ when $\theta$ is in the range between $57^{\circ}$ and $95^{\circ}$ or smaller beads, $d_{p}(\mathrm{~mm})=0.212$ 0.300 and $<0.106$ when $\theta$ is in the range between $68^{\circ}$ and $72^{\circ}$, as shown in Fig. 8 (a) and (b). The whole stack detached from the interface without experiencing wicking for smaller beads, $d_{p}(\mathrm{~mm})=0.212-0.300$ and $<0.106$ when $\theta$ is in the range between $74^{\circ}$ and $93^{\circ}$.

Table 1. Wicking transition in terms of the critical ethanol volume fraction, $\mathrm{V}_{\mathrm{f}}{ }^{*}$ and corresponding contact angle $\theta_{0}{ }^{*}$ for different sizes of beads. The slopes obtained from equation 2 and from the experiments are reported.

\begin{tabular}{|c|c|c|c|c|}
\hline$d_{p}(\mathrm{~mm})$ & $V_{f}^{*}$ & $\theta_{\sigma}^{*}$ & $\begin{array}{c}\text { Model } \\
\text { equation } \\
(2)\end{array}$ & $\begin{array}{c}\text { Fitting } \\
\text { experimental } \\
\text { slope }\end{array}$ \\
\hline $0.75-1.00$ & $0.35-0.40$ & $60.0-75.1$ & -8 & -2.2 \\
\hline$<0.50$ & $0.30-0.35$ & $74.4-76.8$ & -28 & -1.7 \\
\hline $0.212-0.300$ & $0.35-0.40$ & $71.7-71.9$ & -20 & -0.7 \\
\hline$<0.106$ & $0.30-0.35$ & $68.1-74.0$ & -14 & -0.8 \\
\hline 0.104 & 0.73 & 51.0 & -5 & -0.6 \\
\hline
\end{tabular}

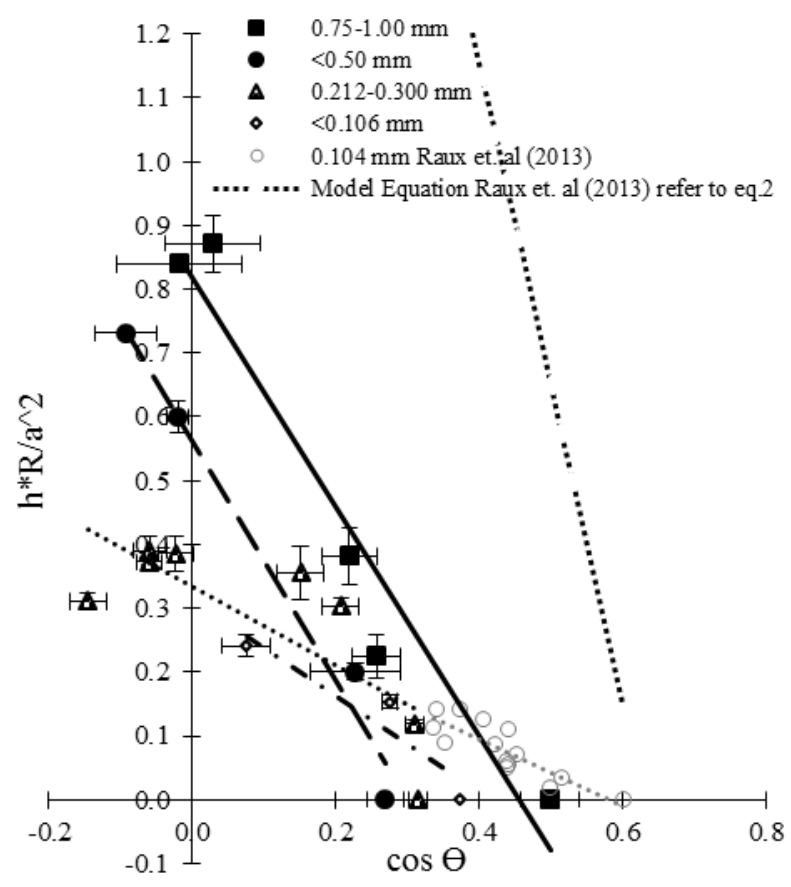

Fig. 7. Normalised critical stack depth vs. the cosine of the bead contact angle. Beads of different diameter are represented using different symbols, as described in the legend.

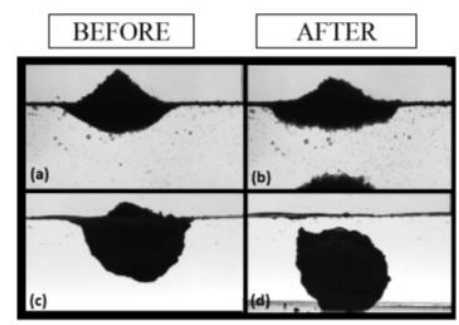

Fig. 8. Images of stacks at $V_{f}=0.15$, (a) - (b) experiencing wicking (for $d_{p}=0.75-1.00 \mathrm{~mm}$ ) and (c) - (d) detaching from the interface (for $d_{p}=0.212-0.300 \mathrm{~mm}$ ). 
The threshold density ratio, $\Delta$, that can be calculated by using equation (1) is in the range between 0.03 and 0.40 , thus always smaller than 1 . Rafts can indeed be built for all the grains and solutions considered, in agreement with Abkarian et al. [21], who proposed that particle rafts would not sink when $\Delta \leq 1$. However, when $\Delta<$ 0.1 , the whole stack was observed to detach from the interface, instead of experiencing wicking.

\subsection{Flow Cell}

The experiments in the flow cell were carried out using two liquid mixture $V_{f}=0.15$ and 0.30 .

The larger bead fractions $d_{p}(\mathrm{~mm})=0.75-1.00$ and $<0.50$ at $\theta=88 \pm 3.8^{\circ}$ and $91 \pm 0.9^{\circ}$ were always found to experience wicking when the agitation is started. As a result of the wicking induced by the liquid flow, the shape of the stack changed from spherical to flat as it can be seen in Fig. 9 (a) to (b). Then, the whole stack detaches from the interface as a cluster, as illustrate in Fig. 9 (c) to (d).

When $V_{f}=0.30$, for all beads, the stack is relatively small and experiences wicking throughout the whole agitation process until the stack disperses, no detachment of stack is observed.

As mentioned above, when $V_{f}<0.30$, which corresponds to $\theta$ is in the range between $74^{\circ}$ and $93^{\circ}$, smaller beads detached from the interface in the static cell, without any agitation. Hence, it was decided to study how shallow stacks with $h<h^{*}$ behave during agitation. For these shallow stack composed of small beads, adding beads onto the stack pulls the layer of grains at the interface (the raft) toward the stack. Fig. 9 (e) to (h) show that when the liquid is flowing, both sides of the raft progressively shrink toward the stack. The flow destabilises the raft and stack, which fall both into the liquid. On the contrary, with larger beads, the liquid agitation was not sufficient to entrain the raft into the liquid.

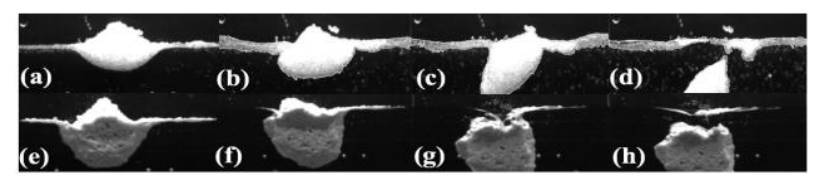

Fig. 9. Images of two different stacks detached from the interface during agitation at $V_{f}=0.15 . d_{p}=0.75-1.00 \mathrm{~mm}$, from (a) to (d) and $d_{p}<0.106 \mathrm{~mm}$, from (e) to (h).

\section{Conclusions}

Two different phenomena have been observed when pouring beads on a static liquid, namely wicking and the detachment of the whole stacks. The critical stack depth on a static liquid decreases with the grain contact angle and when increasing the grain diameter. It was concluded that the difference in stack thickness by forced impregnation is purely due to their particle size and wettability. In agreement with Abkarian et al. [21], wide layers of grains (rafts) can be formed when $\Delta \leq 1$. However, when $\Delta<0.1$, the stacks were detached from the interface, without any wicking. Introducing flow in the liquid can either promote wicking or destabilise the whole stack. In the second case, the whole stack sinks, forming a heterogeneous powder structure that is wet outside and dry inside. This structure is reminiscent of powder lumps: an undesired outcome, when dispersing most consumer good powders, including beverages and detergents. It is suggested that plotting a state diagram to separate wicking and detachment of stack states would provide some highly interesting relationship. As the correlations between the trendline and points in both Fig. 7 are not obvious, this suggests that further exploration of the critical stack depth by using more different contact angles would be valuable. Lastly, more complex grains such as real powders will be considered for use in this study.

\section{References}

1. J. Dupas, L. Forny, and M. Ramaioli, J. Coll. Interface Sci., 448, 51-56 (2015)

2. P. S. Raux, H. Cockenpot, M. Ramaioli, D. Quéré, and C. Clanet, Langmuir, 29, 3636-3644 (2013)

3. J. Dupas, E. Verneuil, M. Ramaioli, L. Forny, L. Talini, and F. Lequeux, Langmuir, 29, 12572-12578 (2013)

4. X. Gao and L. Jiang, Nature, 432, 36 (2004)

5. D. Vella, D. G. Lee, and H. Y. Kim, Langmuir, 22, 5979-5981 (2006)

6. D. Vella and P. D. Metcalfe, Phys. Fluids, 19, 72108 (2007)

7. D. G. G. Lee and H.-Y. Y. Kim, Langmuir, 24, 142145 (2008)

8. J. B. Keller, Phys. Fluids, 10, 3009 (1998)

9. D. G. G. Lee and H.-Y. Y. Kim, Phys. Fluids, 23, 72104 (2011)

10. D. Vella and J. Li, Phys. Fluids, 22, 52104 (2010)

11. P. G. de Gennes, F. Brochard-Wyart, and D. Quere, Capillarity and Wetting Phenomena: Drops, Bubbles, Pearls, Waves (Springer, 2004)

12. E. W. Washburn, Phys. Rev., 17, 273-283 (1921)

13. N. Fries and M. Dreyer, J. Coll. Interface Sci., 320, 259-263 (2008)

14. M. Reyssat, L. Y. Sangne, E. A. van Nierop, and H. A. Stone, EPL Europhys. Lett., 86, 56002 (2009)

15. W. Bin Young, Colloids Surfaces A Physicochem. Eng. Asp., 234, 123-128 (2004)

16. S. Ban, E. Wolfram, and S. Rohrsetzer, Colloids Surfaces, 22, 301-309 (1987)

17. C. A. E. Hamlett et al., Environ. Sci. Technol., 45, 9666-9670 (2011)

18. N. J. Shirtcliffe, G. McHale, M. I. Newton, F. B. Pyatt, and S. H. Doerr, Appl. Phys. Lett., 89, 94101 (2006)

19. S. G. Jones, N. Abbasi, A. Ahuja, V. Truong, and S. S. H. Tsai, Phys. Fluids, 27, 72102 (2015)

20. D. Vella and L. Mahadevan, Am. J. Phys., 73, 817 (2005)

21. M. Abkarian, S. Protière, J. M. Aristoff, and H. A. Stone, Nature. Commun., 4, 1895 (2013)

22. D. Vella, P. Metcalfe, and R. Whittaker, J. Fluid Mech., 549, 215-224 (2005) 